\title{
Ghost-gluon and ghost-quark bound states and their role in BRST quartets
}

\author{
Natalia Alkofer* \\ Institut für Physik, Karl-Franzens-Universität, Universitätsplatz 5, A-8010 Graz, Austria \\ E-mail: natalia.alkofereedu.uni-graz.at
}

\section{Reinhard Alkofer}

Institut für Physik, Karl-Franzens-Universität, Universitätsplatz, 5, A-8010 Graz, Austria

E-mail: reinhard.alkofer@uni-graz.at

\begin{abstract}
A non-perturbative version of the BRST quartet mechanism in infrared Landau gauge QCD is proposed for transverse gluons and quarks. Based on the positivity violation for transverse gluons the content of the respective non-perturbative BRST quartet is derived. To identify the gluon's BRSTdaughter and second parent state, a truncated Bethe-Salpeter equation for the gluon-(anti-)ghost bound state is investigated. We comment shortly on several equivalent forms of this equation. Repeating the same construction for quarks leads to a truncated Bethe-Salpeter equation for a fundamentally charged quark-(anti-)ghost bound state. It turns out that a cardinal input to this equation is given by the fully dressed quark-gluon vertex, and that it is indispensable to dress the quark-gluon vertex in this equation in order to obtain a consistent truncation.
\end{abstract}

International Workshop on QCD Green's Functions, Confinement and Phenomenology 5-9 September 2011

Trento, Italy

\footnotetext{
* Speaker.
} 


\section{Introduction}

The topic of this workshop is QCD Green's functions as well as their relation to confinement and the corresponding phenomenology. Amongst these Green's functions the gluon propagator is one of (if not the) most investigated one. It has been calculated with various methods in various gauges. In Landau gauge, where all gluons contributing to physical states are strictly transverse and therefore in the massless representation of the Poincare group, it is described by one renormalisation function $Z\left(k^{2}\right)$. Already in the late seventies it has been noted that there is a conflict between antiscreening ${ }^{1}$ by and positivity of transverse gluons [1]. Nowadays, thirty years later, there is no doubt any more that the gluon propagator of Landau gauge QCD violates positivity, see e.g. Ref. [2] and references therein. Due to this property we can conclude that the one-gluon-state (with a transverse gluon) belongs to the states of negative norm in the state space of Landau gauge QCD. This is in contrast to the characterization of the indefinite-metric state space of gauge theories based on perturbation theory (or, expressed more formally, on the limit of vanishing gauge coupling): then only the longitudinal and the time-like component of the gauge boson (as well as ghosts and antighosts) are related to the negative and zero norm states [3, 4]. One possible route is then to proceed by establishing a Hilbert space of Becchi-Rouet-Stora-Tyutin (BRST) singlets via the construction of a BRST cohomology, see e.g. [5, 4].

It is evident that in a full solution to Landau gauge QCD in this way all colour charged particles will be removed from the physical spectrum because the BRST singlets of gauge-fixed theory are gauge-invariant states. Note that this construction does not tell us how the removal of non-invariant states occurs dynamically, it is even up to today unclear how the details of a non-perturbative BRST cohomology might work. Within the context of Landau gauge QCD we formulated some first steps into this direction $[6]^{2}$. The essential point to be noted is the way the non-perturbative character of the BRST multiplets containing the transverse gluon, resp., the quark is realized: These quartets are besides the transverse gluon, resp., quark build of bound states. It is here where the full difficulty on non-Abelian gauge theory (and therefore QCD) strikes back: The solution of relativistic bound state equations in a symmetry-preserving way is a notoriously complicated problem.

As will be detailed below the above remarks on positivity violation in the gluon propagator especially imply that the quantum state of one transverse gluon can be identified with a parent state in a BRST quartet. As we stated above the other members, however, have to be non-perturbative, i.e. bound states. We will present the identification of possible members of this quartet and describe a strategy to provide evidence for their role in the formalism of covariantly gauge-fixed Yang-Mills (YM) theory. If this approach turns out to be successful it will provide further details on the kinematical aspects of gluon confinement in Landau gauge.

In the quark sector of Landau gauge QCD the situation is even much less clear. To the best of our knowledge there is no convincing evidence on the issue of positivity violation for the quark propagator in either direction, positivity-violating or positivity-respecting. Following a similar strategy as for the gluons may allow a clarification whether quarks are also positivity violating. Therefore, we will present also the case of constructing a (hypothetical) quark BRST quartet.

\footnotetext{
${ }^{1}$ leading to the celebrated asymptotic freedom property

${ }^{2}$ For a presentation of this topic in a more introductory style we refer to [7].
} 


\section{Non-perturbative BRST quartets in Landau gauge QCD}

A basic understanding of the BRST quartet cancellation mechanism can be gained from analysing the so-called elementary quartet $[5,4]$. Referring to the gauge fields generically as gluons ${ }^{3}$ the elementary quartet consists of longitudinal and time-like gluons as well as Faddeev-Popov (FP) ghosts and antighosts ${ }^{4}$. We start with the definition of the BRST transformation $\delta_{B}:{ }^{5}$

$$
\begin{array}{ll}
\delta_{B} A_{\mu}^{a}=\widetilde{Z}_{3} D_{\mu}^{a b} c^{b} \lambda, & \delta_{B} q=-i g t^{a} \widetilde{Z}_{1} c^{a} q \lambda, \\
\delta_{B} c^{a}=-\frac{g}{2} f^{a b c} \widetilde{Z}_{1} c^{b} c^{c} \lambda, & \delta_{B} \bar{c}^{a}=B^{a} \lambda,
\end{array}
$$

where $D_{\mu}^{a b}$ is the covariant derivative. The Grassmann parameter $\lambda$ carries ghost number $N_{\mathrm{FP}}=-1$. $\widetilde{Z}_{1}$ and $\widetilde{Z}_{3}$ are the ghost-gluon-vertex and the ghost wave function renormalisation constants.

The BRST charge operator $Q_{B}$ (e.g., derived via Noether's theorem) provides then the BRST transform of a field, for example $Q_{B} A_{\mu}^{a}=\widetilde{Z}_{3} D_{\mu}^{a b} c^{b}$. It is straightforward to prove that $Q_{B}$ is nilpotent, $Q_{B}^{2}=0$. In addition, as $Q_{B}$ carries ghost number, its commutator with the ghost number operator $Q_{c}$ is given by $\left[i Q_{c}, Q_{B}\right]=Q_{B}$.

In the limit of vanishing coupling $g \rightarrow 0$, the elementary BRST quartet can be read from the BRST transformation in the following way: The forward-polarized gluon ( $c f$. p. 511 of Ref. [3]) is transformed via $Q_{B}$ into a ghost. Note that for $g \rightarrow 0$ the one-ghost-state is annihilated by the BRST transformation. The FP-conjugated state of the ghost, the antighost, is mapped by $Q_{B}$ to a state of the field $B^{a}$ whose states are exactly the backward-polarized gluons. Forward-polarized gluons and antighosts are BRST non-singlets (and have negative norm), the backward-polarized gluons and ghosts are in the image of $Q_{B}$, a property which implies that they have zero norm. On the other hand, there are non-vanishing off-diagonal matrix elements, and they are usually for convenience normalized to one. It is this algebraic structure which guarantees that there are no non-vanishing $S$-matrix elements between BRST singlets and BRST quartets.

The elementary quartet can serve to explicate the notation of Refs. [8, 5]: We call the negative norm state we start as 1st parent, its BRST transform 1st daughter. The FP-conjugated state of the latter is then named 2nd parent, the corresponding BRST transform 2nd daughter.

The corresponding construction of the BRST quartet starting from a transverse gluon, resp., a quark is displayed in Fig. 1. Starting from the first transformation in eq. (2.1) and noting already that the term with the ghost field alone is "used up" for the elementary quartet, it is obvious that the field content of the 1st daughter state is of the form $\widetilde{Z}_{3} g f^{a b c} A_{\mu}^{c} c^{b}$. Following the construction the 2nd parent is $\widetilde{Z}_{3} g f^{a b c} A_{\mu}^{c} \bar{c}^{b}$. Its BRST transform, the 2nd daughter, is then $-\widetilde{Z}_{3}^{2} g f^{a b c} \bar{c}^{b} D_{\mu}^{c d} c^{d}+$ $\widetilde{Z}_{3} g f^{a b c} B^{b} A_{\mu}^{c}$. The field content for the quark BRST quartet accordingly reads:

$$
q, \quad-\widetilde{Z}_{1} i g t^{a} c^{a} q, \quad-\widetilde{Z}_{1} i g t^{a} \bar{c}^{a} q, \quad-\widetilde{Z}_{1} i g t^{a} B^{a} q-\widetilde{Z}_{1}^{2} g^{2} \frac{i}{2} f^{a b c} t^{c} \bar{c}^{a} c^{b} q .
$$

For every "one-transverse-gluon" state, resp., "one-quark" state there should occur exactly one degenerate daughter state. This necessitates the existence of a ghost-gluon bound state in the adjoint and of a quark-ghost bound state in the fundamental representation [6, 7]. The FP-charge reversed

\footnotetext{
${ }^{3}$ Of course, this procedure applies to all YM theories in Faddeev-Popov quantization.

${ }^{4}$ One cannot consider directly the longitudinal and time-like gluons but needs to define linear superpositions of them, the forward, resp., backward polarized gluons, see e.g. Chapter 16 of Ref. [3] for a definition of these states.

${ }^{5}$ The nilpotency of the BRST transformation is explicit in a representation with Nakanishi-Lautrup field $B^{a}$. It becomes on-shell identical to the gauge fixing condition, $B^{a}=(1 / \xi) \partial_{\mu} A_{\mu}^{a}$, with $\xi$ being the gauge parameter.
} 


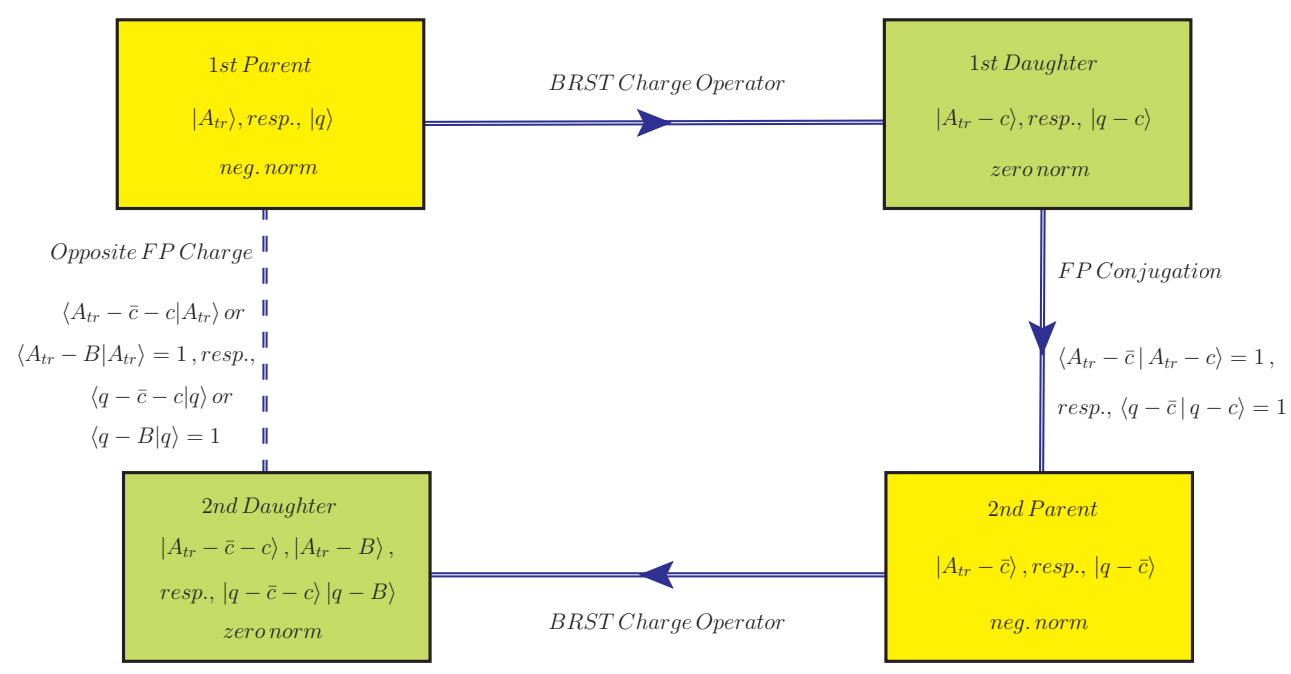

Figure 1: An illustration of the construction of the BRST quartets of the transverse gluon, resp., quark.

2nd parent state is an antighost-gluon, resp., antighost-quark bound state. Landau gauge provides now an advantage: In the limit $\xi \rightarrow 0$ the formalism becomes ghost-antighost-symmetric, and thus a ghost-gluon (-quark) bound state implies a degenerate antighost-gluon (-quark) bound state with same quantum numbers. The BRST transformation leaves then three, resp., two possibilities for the 2nd daughter. In the gluon case they are ghost-antighost, ghost-antighost-gluon, or a bound state of two differently polarized gluons, whereas in the quark case it is a ghost-antighost-quark, or a bound state of the backward-polarized gluon with the quark.

At this point a remark with respect to a fundamental difference between transverse gluons and quarks is in order. As stated already above the transverse gluons, as vector states with two polarizations, are in the massless representation of the Poincare group. ${ }^{6}$ Correspondingly all the other partners of the quartet will appear with two polarizations and are thus also in the massless representation of the Poincare group. Quarks, on the other hand, will be described by massive Dirac fermions. For light quarks mostly dynamical chiral symmetry breaking and for heavy quarks mostly explicit chiral symmetry breaking determines the corresponding effective masses. In case the quark BRST quartet exists its members will be all, in the sense of the representation of the Poincare group, massive Dirac fermions. However, contrary to the situation for the transverse gluons the question of positivity violation of quarks is unresolved. Although the infrared behaviour of the quark propagator comes out almost identical in all the studies done so far its analytic structure remains elusive. In functional approaches this can be traced back to the fact that the analytic structure of the quark propagator is highly sensitive to details in the quark-gluon vertex, see, e.g., Ref. $[13,14]$. The quark-gluon vertex is, on the other hand, also very strongly influenced by dynamical and/or explicit chiral symmetry breaking [15, 16, 17]. Already these remarks make evident that the mass generation for quarks related to chiral symmetry breaking depends strongly on details of the dynamics. What kind of mechanism then guarantees that the corresponding BRST

\footnotetext{
${ }^{6}$ Please note that this does not exclude the kind of screening mass determined by a non-vanishing infrared value of the gluon propagator. Such a "gluon screening mass" has been suggested already thirty years ago in Ref. [9] and has been found more recently, e.g., in Refs. [10, 11, 12].
} 
bound states are degenerate with the quark states is completely unknown. In the following we will have a closer look to the bound state equations for the non-perturbative BRST quartet states in the hope that their structure provides an insight into possible answers to these questions.

\section{Ghost-gluon bound state equation}

Our aim is to derive a consistently truncated bound state equation for the 1st daughter of the BRST quartet of the transverse gluon. As stated above this gives us automatically the bound state equation for the 2nd parent. Determining the necessary input in terms of primitively divergent YM Green's functions is certainly a demanding task. However, given the knowledge acquired for the gluon and ghost propagators as well as for the gluon-ghost and three-gluon vertex functions over the last decade, a reasonable precise modelling of this input is available. Therefore the real challenge is to find a trustable truncation.

To this end we exploit a peculiarity of the solutions of Dyson-Schwinger and Functional Renormalisation Group equations: There is one unique scaling solution with power laws for the Green's functions and an one-parameter family of solutions, the so-called decoupling solutions. The latter are infrared trivial solutions which possess as an endpoint exactly the scaling solution characterized by infrared power laws. Numerical solutions of the decoupling type (there called "massive solution") have been published in $[10,11]$ and references therein. A recent detailed description and comparison of these two types of solutions has been given in Ref. [12], see also Refs. $[18,19,20,21,22]$. This leads to the idea to order the diagrams according to the infrared behaviour seen in the scaling solution. If the conjecture of Ref. [23], namely that the occurrence of different types of solutions is a gauge artefact, is correct, it is sufficient that only one non-perturbative completion of Landau gauge with scaling solution exists to make the above described truncation well-founded. Second, one may argue based on continuity that diagrams which are leading in the scaling solution will be numerically dominant in the decoupling solution.

Investigations of the scaling solution have been performed for the gluon and ghost propagators [24, 25, 26, 27, 28] and for Yang-Mills vertex functions [29, 30, 31, 32]. In this solution every oneparticle irreducible Green's functions behaves like a power-law in the deep infrared. Therefore one can attribute an infrared exponent to every diagram appearing in a Dyson-Schwinger or Functional Renormalisation Group equation. With this in mind the ghost-gluon bound state is looked for in the ghost-gluon scattering kernel. To this end we want to truncate this quantity to the infrared leading term. We use DoDSE [33, 34, 35] to derive the diagrammatic expressions for the Dyson-Schwinger equation of this four-point function. A diagram-by-diagram infrared power counting is performed by attributing anomalous infrared exponents to each of them. A consistency check is provided by the fact that the a priori known infrared exponent of the ghost-gluon scattering kernel is refound.

To select the diagrams to be kept in the bound state equation we require: They should contain the one-particle irreducible ghost-ghost-gluon-gluon four-point function and no $n \geq 5$-point function, they should be infrared leading, and the interaction shall take place in the ghost-gluon channel. This leaves from the many diagrams in the equation for the ghost-gluon scattering kernel only two: One with two ghost and one gluon propagators on internal lines. This is effectively a ghost exchange. And another one with two gluon and one ghost propagators on internal lines. This 

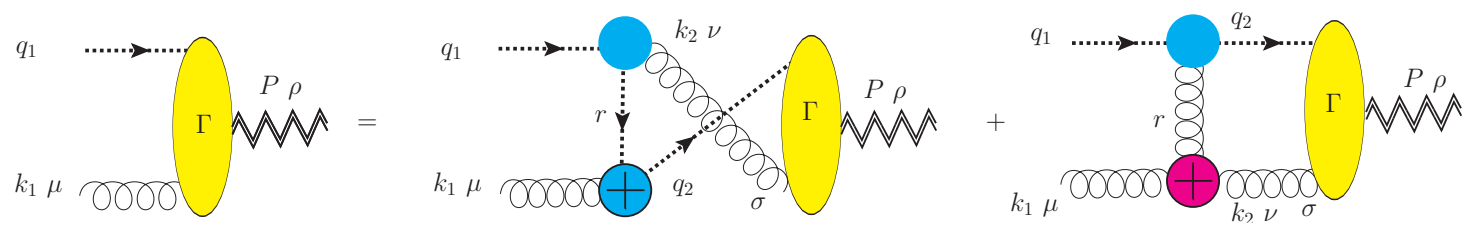

Figure 2: Graphical representation of the ghost-gluon Bethe-Salpeter equation. Crosses denote dressed vertices.

is a gluon exchange. This diagram is also infrared leading because in the scaling solution the fully dressed three-gluon vertex is infrared divergent.

Assuming the existence of a bound state as well as employing the usual decomposition of the (ghost-ghost-gluon-gluon) four-point function into Bethe-Salpeter amplitudes and performing the expansion around the pole (see e.g. Sect. 6.1 of Ref. [36]) one arrives at the Bethe-Salpeter equation depicted in Fig. 2. Using the propagator parameterizations of e.g. Ref. [13], the ghost-gluon vertex of Ref. [37], and the three-gluon vertex of Ref. [38], one can derive a self-consistent equation for the corresponding Bethe-Salpeter amplitude containing otherwise only known quantities.

As stated above, and as explicitly demonstrated in ref. [6], the Bethe-Salpeter amplitude for this bound state in the adjoint colour representation is transverse, i.e., this bound state is as expected in the massless representation of the Poincare group. Restricting oneself to the (presumably dominant) ghost exchange and going to the limit of soft momenta, in ref. [6] the following equation for the ghost-gluon Bethe-Salpeter amplitude (being described by one function $F$ as only the transverse component exists) is obtained:

$$
F\left(k_{1}^{2}\right)=\widetilde{Z}_{3}^{2} g^{2} N_{c}^{2} \int \frac{d^{4} k_{2}}{(2 \pi)^{4}} \frac{G\left(\left(k_{1}+k_{2}\right)^{2}\right)}{\left(k_{1}+k_{2}\right)^{2}} \frac{G\left(k_{2}^{2}\right)}{k_{2}^{2}} \frac{Z\left(k_{2}^{2}\right)}{k_{2}^{2}} \frac{1}{3}\left(k_{1} \cdot k_{2}\right)\left(1-\frac{\left(k_{1} \cdot k_{2}\right)^{2}}{k_{1}^{2} k_{2}^{2}}\right) F\left(k_{2}^{2}\right),
$$

where $Z$ and $G$ are the gluon and ghost renormalisation function, respectively. Note that the product $G^{2} Z$ appearing in the integrand is a renormalisation group invariant [39]. Using an alternative projection on Lorentz indices one can derive an equivalent, somewhat simpler, equation:

$$
F\left(k_{1}^{2}\right)=-\frac{1}{3} k_{1}^{2} Z_{3}^{2} g^{2} N_{c}^{2} \int \frac{d^{4} k_{2}}{(2 \pi)^{4}} \frac{G\left(\left(k_{1}+k_{2}\right)^{2}\right)}{\left(k_{1}+k_{2}\right)^{2}} \frac{G\left(k_{2}^{2}\right)}{k_{2}^{2}} \frac{Z\left(k_{2}^{2}\right)}{k_{2}^{2}}\left(1-\frac{\left(k_{1} \cdot k_{2}\right)^{2}}{k_{1}^{2} k_{2}^{2}}\right) F\left(k_{2}^{2}\right) .
$$

Attempts to numerically solve these equations by iteration with the input as described above have generated curves which deviate from each other in the infrared. Also for both curves some unexpected features in deep infrared occur. Therefore we decided to change the numerical method, work in this direction is in progress.

\section{Ghost-quark bound state equation}

The quark propagator is due to dynamical or explicit chiral symmetry breaking infrared finite. In the scaling solution, the twelve possible Dirac tensor structures of the quark-gluon vertex are then all infrared divergent. This leads to an $1 / k^{4}$ behaviour of the kernel in the four-quark function, $k$ being the momentum exchange. This is indicative of a linearly rising potential between static quarks, and thus quark confinement. 

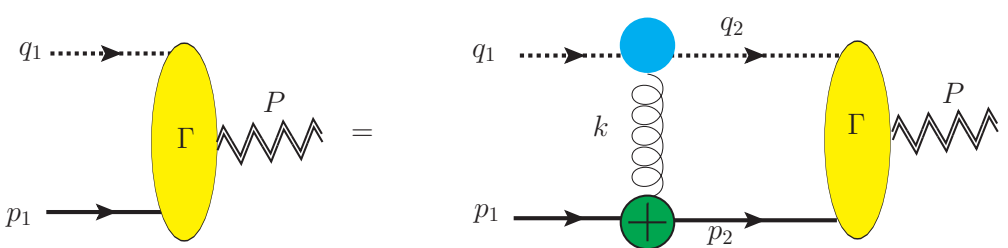

Figure 3: Graphical representation of the ghost-quark Bethe-Salpeter equation. Here the quark-gluon vertex is fully dressed.

Using the same truncation requirements and the same derivation of the Bethe-Salpeter equation as in the previous subsection one arrives at the equation depicted in Fig. 3 [6]. This equation is in full agreement with the infrared analysis of the scaling solution. In its kernel the anomalous infrared exponents cancel if and only if the fully dressed quark-gluon vertex is taken into account. Again this is in agreement with the expectations arising from previous studies.

\section{Outlook}

In this work we reported on a suggestion how to study the non-perturbative BRST quartets generated by transverse gluons and quarks quantitatively. Attempts of a numerical solution of the ghost-gluon Bethe-Salpeter equation uncovered so far only its sensitivity to the deep infrared. Within this project many open questions still remain: What are the bound states representing the respective 2nd daughters? Is BRST dynamically broken? Can we answer in this way the question of positivity or positivity violation for quarks? And does all this relate to quark confinement?

\section{Acknowledgments}

We thank the organizers of $Q C D-T N T-I I$, and especially Daniele Binosi and Joannis Papavassiliou, for all their efforts which made this highly interesting workshop possible. The figures here have been drawn with JaxoDraw [40, 41], we want to take the opportunity to thank the authors.

\section{References}

[1] R. Oehme and W. Zimmermann, Phys. Rev. D 21, 471 (1980).

[2] P. O. Bowman et al., Phys. Rev. D 76 (2007) 094505 [arXiv:hep-lat/0703022].

[3] M. E. Peskin and D. V. Schroeder, "An Introduction To Quantum Field Theory," Reading, USA: Addison-Wesley (1995) $842 p$.

[4] S. Weinberg, "The quantum theory of fields. Vol. 2: Modern applications," Cambridge, UK: Univ. Pr. (1996) $489 p$.

[5] N. Nakanishi and I. Ojima, World Sci. Lect. Notes Phys. 27 (1990) 1.

[6] N. Alkofer and R. Alkofer, Phys. Lett. B 702 (2011) 158 [arXiv:1102.2753 [hep-th]].

[7] N. Alkofer and R. Alkofer, PoS FACESQCD (2011) 043 [arXiv:1102.3119 [hep-th]].

[8] T. Kugo and I. Ojima, Prog. Theor. Phys. Suppl. 66, 1 (1979). 
[9] J. M. Cornwall, Phys. Rev. D 26 (1982) 1453.

[10] A.C. Aguilar, D. Binosi, J. Papavassiliou, Phys. Rev. D78 (2008) 025010 [arXiv:0802.1870 [hep-ph]].

[11] P. Boucaud et al., JHEP 0806 (2008) 099 [arXiv: 0803.2161 [hep-ph]].

[12] C.S. Fischer, A. Maas and J.M. Pawlowski, Ann. Phys. 324 (2009) 2408 [arXiv:0810.1987 [hep-ph]].

[13] R. Alkofer et al., Phys. Rev. D70 (2004) 014014 [hep-ph/0309077]; Nucl. Phys. Proc. Suppl. 141 (2005) 122 [hep-ph/0309078].

[14] A. C. Aguilar and J. Papavassiliou, Phys. Rev. D 83, 014013 (2011) [arXiv:1010.5815 [hep-ph]].

[15] J. I. Skullerud et al., JHEP 0304 (2003) 047 [arXiv:hep-ph/0303176].

[16] R. Alkofer, C. S. Fischer and F. J. Llanes-Estrada, Mod. Phys. Lett. A23 (2008) 1105 [arXiv:hep-ph/ 0607293].

[17] R. Alkofer et al., Annals Phys. 324 (2009) 106 [arXiv: 0804.3042 [hep-ph]].

[18] R. Alkofer, M. Q. Huber, K. Schwenzer, Phys. Rev. D 81 (2010) 105010 [arXiv:0801.2762 [hep-th]].

[19] M. Q. Huber, K. Schwenzer and R. Alkofer, Eur. Phys. J. C68 (2010) 581 [arXiv:0904.1873 [hep-th]]; PoS FACESQCD (2011) 001 [arXiv:1103.0236 [hep-th]].

[20] A. P. Szczepaniak and E. S. Swanson, Phys. Rev. D 65 (2002) 025012 [arXiv:hep-ph/0107078].

[21] D. Epple et al., Phys. Rev. D 77 (2008) 085007 [arXiv:0712.3694 [hep-th]].

[22] C. S. Fischer and J. M. Pawlowski, Phys. Rev. D 80 (2009) 025023 [arXiv:0903.2193 [hep-th]].

[23] A. Maas, Phys. Lett. B 689 (2010) 107 [arXiv:0907. 5185 [hep-lat]].

[24] L. von Smekal, R. Alkofer and A. Hauck, Phys. Rev. Lett. 79 (1997) 3591 [arXiv:hep-ph/9705242].

[25] P. Watson and R. Alkofer, Phys. Rev. Lett. 86 (2001) 5239 [arXiv:hep-ph/0102332].

[26] D. Zwanziger, Phys. Rev. D 65 (2002) 094039 [arXiv:hep-th/0109224].

[27] C. Lerche and L. von Smekal, Phys. Rev. D 65 (2002) 125006 [arXiv:hep-ph/0202194].

[28] C. S. Fischer and R. Alkofer, Phys. Lett. B 536 (2002) 177 [arXiv:hep-ph/0202202].

[29] R. Alkofer, C. S. Fischer and F. J. Llanes-Estrada, Phys. Lett. B 611 (2005) 279 [arXiv:hep-th/0412330].

[30] M. Q. Huber et al., Phys. Lett. B 659 (2008) 434 [arXiv:0705.3809 [hep-ph]].

[31] R. Alkofer et al., Proceedings of the conference Confinement 9 [arXiv:1011.5831 [hep-ph]].

[32] R. Alkofer, PoS FACESQCD (2011) 030 [arXiv:1102.3166 [hep-th]].

[33] R. Alkofer, M.Q. Huber and K. Schwenzer, Comput. Phys. Commun. 180 (2009) 965 [arXiv:0808.2939 [hep-th]].

[34] M. Q. Huber, Ph.D. Thesis, University Graz, 2010 [arXiv:1005.1775 [hep-th]].

[35] M. Q. Huber and J. Braun, arXiv:1102.5307 [hep-th].

[36] R. Alkofer and L. von Smekal, Phys. Rept. 353 (2001) 281 [arXiv:hep-ph/0007355].

[37] W. Schleifenbaum et al., Phys. Rev. D 72 (2005) 014017 [arXiv:hep-ph/0411052].

[38] R. Alkofer, M. Q. Huber, K. Schwenzer, Eur. Phys. J. C 62 (2009) 761 [arXiv:0812.4045 [hep-ph]].

[39] R. Alkofer, C. S. Fischer and L. von Smekal, Acta Phys. Slov. 52 (2002) 191 [arXiv:hep-ph/0205125].

[40] D. Binosi and L. Theussl, Comput. Phys. Commun. 161 (2004) 76 [arXiv:hep-ph/0309015].

[41] D. Binosi et al., Comput. Phys. Commun. 180 (2009) 1709 [arXiv:0811.4113 [hep-ph]]. 